\title{
PI6-17. Antigen sensitivity is a major determinant of CD8+ T-cell polyfunctionality and HIV suppressive activity
}

\author{
J Almeida, D Sauce, D Price, L Papagno, S Shin, A Moris, M Larsen, \\ G Pancino, D Douek, B Autran, A Saez-Cirion and V Appay*
}

Address: INSERM U945, Paris, France

* Corresponding author

from AIDS Vaccine 2009

Paris, France. 19-22 October 2009

Published: 22 October 2009

Retrovirology 2009, 6(Suppl 3):P246 doi: I0.I I86/I 742-4690-6-S3-P246

This abstract is available from: http://www.retrovirology.com/content/6/S3/P246

(c) 2009 Almeida et al; licensee BioMed Central Ltd.

\section{Background}

Induction of effective CD8+ T cells remains a major goal of vaccine strategies against HIV. Recent work suggests that polyfunctionality and HIV suppressive activity are associated with CD8+ T cell efficacy in HIV infected patients, so that generation of CD8+ T cells with such attributes through vaccination is considered highly desirable. However, the precise determinants of such qualitative attributes remain actually poorly understood, which is a clear drawback for rationale vaccine design.

\section{Methods}

In order to provide clear insights into the nature of effective CD8+ T cells, we performed a thorough study of CD8+ $\mathrm{T}$ cell attributes associated with efficacy in HIV infection at the clonal level. Isolated CD8+ T clonotypes were expanded in vitro and their functional attributes assessed using polychromatic flow cytometry and HIV suppression assays. Observations were confirmed for the CD4+ T cell lineage as well as with ex vivo data from HIV infected donors.

\section{Results}

We show here that $\mathrm{T}$ cell polyfunctionality is based on a hierarchical induction of distinct functions according to $\mathrm{T}$ cell antigen sensitivity, or recognition efficiency, which defines the strength of interaction between the effector cell and its target. Overall, polyfunctionality and HIV suppressive activity appear to be directly linked to the level of antigen sensitivity. Highly sensitive CD8+ T cells display polyfunctional profiles, efficient proliferation and potent HIV suppressive activity.

\section{Conclusion}

Antigen sensitivity emerges as a key determinant of CD8+ $T$ cell efficacy in HIV infection. These findings emphasize the importance to focus the design and immunomonitoring of candidate vaccines on the induction of T cells with high antigen sensitivity. 